\title{
Higher order effects in electromagnetic dissociation of neutron halo nuclei
}

\author{
S. Typel \\ National Superconducting Cyclotron Laboratory, Michigan State University, East Lansing, Michigan 48824-1321 \\ G. Baur \\ Institut für Kernphysik, Forschungszentrum Jülich, D-52425 Jülich, Germany \\ (Received 16 January 2001; published 22 June 2001)
}

\begin{abstract}
We investigate higher order effects in electromagnetic excitation of neutron halo nuclei using a zero-range model for the neutron-core interaction. In the sudden (or Glauber) approximation all orders in the target-core electromagnetic interaction are taken into account. Small deviations from the sudden approximation are readily calculated. We obtain very simple analytical results and scaling laws for the next-to-leading order effects, which have a simple physical interpretation. For intermediate energy electromagnetic dissociation, higher order effects are generally small. We apply our model to Coulomb dissociation of ${ }^{19} \mathrm{C}$ at $67 \mathrm{~A} \mathrm{MeV}$ and of ${ }^{11} \mathrm{Be}$ at $72 \mathrm{~A} \mathrm{MeV}$. The analytical results are compared to numerical results from the integration of the time-dependent Schrödinger equation. Good agreement is obtained. We conclude that higher order electromagnetic effects are well under control.
\end{abstract}

DOI: 10.1103/PhysRevC.64.024601

PACS number(s): 25.70.De, 25.70.Mn, 25.60.-t

\section{INTRODUCTION}

Electromagnetic excitation of high energy radioactive beams is a powerful method to study electromagnetic properties of loosely bound neutron rich nuclei. For example, the low lying $E 1$ strengths of one-neutron halo nuclei like ${ }^{11} \mathrm{Be}$ and ${ }^{19} \mathrm{C}$ have been studied in this way $[1-3]$. In a similar way, two-neutron halo nuclei like ${ }^{6} \mathrm{He}$ and ${ }^{11} \mathrm{Li}$ were studied. Such experiments are usually analyzed theoretically in first order electromagnetic perturbation theory or the equivalent photon method. In this way, the multipole (especially dipole) strength distribution is obtained. Such an analysis depends on the dominance of first order excitations. Various methods have been developed in order to consider deviations from first order perturbation theory with the usual multipole expansion of the interaction. However, a consistent picture of the importance of these approximations has still not emerged.

By "higher order effects" we mean only electromagnetically induced effects on the relative momentum of the fragments. They can be studied in the semiclassical approximation. In the widest sense all effects that give rise to a deviation from the result of the traditional semiclassical first order perturbative calculation of Coulomb breakup can be summarized under this expression. In the perturbative approach higher order effects can be described as the exchange of more than one photon between the target and the projectile system. "Postacceleration" is also a higher order effect. In a classical picture it can be understood as a different acceleration of the fragments in the Coulomb field of the target which will change both the c.m. momentum and the relative momentum of the particles in the final state. In our calculations we will not treat quantal effects like diffraction or contributions to the breakup from the nuclear interaction.

There are mainly two different approaches for the investigation of higher order effects. In the semiclassical description the projecile moves on a classical trajectory (which is usually well justified) and experiences a time-dependent per- turbation from the target. Only the excitation of the projectile is treated quantally. In contrast to that, the total system of target, projectile, and fragments, respectively, can be described by suitable wave functions in a fully quantum mechanical approach. Each of these approaches has its merits, but, at the same time, can limit the study of certain higher order effects or make it difficult to extract them by a comparison to a suitable first order calculation.

The breakup of the prototype of a loosely bound nucleus, the deuteron, has for a long time been studied in the postform distorted-wave Born approximation (DWBA) theory. Later, it was also applied to neutron halo (core + neutron) nuclei like ${ }^{11} \mathrm{Be}$. In this approach, the Coulomb interaction between the target and the core is taken into account to all orders. This is done by using full Coulomb wave functions in the initial and final states. For a recent review with further references see [4]. A so-called adiabatic breakup theory has recently been developed in [5]. This model is related to the postform DWBA. It leads to a very similar formula; however, the physical interpretation is somewhat different [4]. Without entering into the differences of the two approaches, it is clear that in these theories higher order effects in the Coulomb interaction are automatically included to all orders. It is therefore very interesting to note that Tostevin [6] claims to have found substantial higher order effects in the Coulomb breakup of ${ }^{19} \mathrm{C}[3]$. He compared his results from the adiabatic approach to the one using semiclassical first order theory. In the zero-range limit for the neutron-core interaction both theories agree very well, suggesting that higher order effects are small. Using a finite-range interaction where the neutron is in a bound $2 s_{1 / 2}$ state, both calculations still give very similar relative energy spectra, but they differ by about 35\% in absolute magnitude. Therefore one might conclude that higher order effects are strongly dependent on the internal structure of the halo nucleus.

It is the purpose of this paper to investigate higher order effects in the electromagnetic excitation of neutron halo nuclei by comparing lowest order and higher order approxima- 
tions within the same model for the excitation process using both a zero-range and a finite-range model for the halo nucleus. This is expected to give more reliable statements about the importance of these effects than the comparison of higher order calculations in one theory with first order calculations in another theory, where, e.g., the finite-range effects are treated in another way or (probably of less importance in the present context) the semiclassical approximation is not applied. We will limit ourselves to the semiclassical description, considering only the Coulomb interaction, and will not investigate nuclear induced effects. In our approach we use a classical trajectory to describe the relative motion between the target and the projectile. It should be kept in mind that there is some ambiguity in the definition of this trajectory. The energy loss should be small compared to the total kinetic energy of the projectile and some averaging procedure can be used. We assume that the c.m. of the projectile moves on the classical trajectory (straight line or Rutherford). It has been argued that the electromagnetic interaction of the target affects only the charged core of the projectile; therefore the c.m. of the core has been used for describing the classical motion. However, for intermediate energy this effect was found to be quite small in numerical calculations in [7]. Actually, the result of a first order $E 1$ calculation does not depend on this choice, since the dipole moment of the system does not change. There is only a change of the quadrupole moment but this has small effects since the E2 contribution to the breakup is rather small (see below). Since the c.m. trajectory is fixed, only higher order effects in the relative motion of the fragments can be handled in the semiclassical approach. Since the total momentum of the fragments is much larger than the relative momentum between them, higher order effects have a much larger effect on the relative momentum.

To some approximation the nuclear structure of neutron halo nuclei can be described by rather simple wave functions. Using these wave functions the reaction mechanism can be studied in a very transparent way and analytical results are obtained. In a later stage more refined descriptions of the nuclei can be introduced. We recall some results from [8] and apply the model to the electromagnetic breakup of ${ }^{19} \mathrm{C}$ and ${ }^{11} \mathrm{Be}$ in comparison to more accurate descriptions. In Sec. II the theoretical framework is given; results and comparison to experimental results [3] are presented in Sec. III. Conclusions are given in Sec. IV.

\section{THEORETICAL FRAMEWORK}

We follow very closely the approach of [8]; see also [9]. In this straight-line semiclassical model a projectile with charge $+Z e$ impinges on a neutron + core $(n+c)$ system with impact parameter $b$ and velocity $v$. The ground state wave function of the bound $n+c$ system is given by a simple Yukawa type wave function

$$
\phi=\sqrt{\frac{\eta}{2 \pi}} \frac{\exp (-\eta r)}{r},
$$

where the parameter $\eta$ is related to the binding energy $E_{0}$ by
$E_{0}=\hbar^{2} \eta^{2} / 2 m$ with the reduced mass of the system $m$ $=m_{n} m_{c} /\left(m_{n}+m_{c}\right)$. The final continuum state is given by

$$
\phi_{q}^{(-)}=\exp (i \vec{q} \cdot \vec{r})-\frac{1}{\eta-i q} \frac{\exp (-i q r)}{r},
$$

where the wave number $q$ is related to the relative energy by $E_{\text {rel }}=\hbar^{2} q^{2} / 2 m$. With these wave functions the breakup probability can be calculated analytically in the sudden approximation (corresponding to the Glauber or frozen nucleus approximation) of semiclassical Coulomb excitation theory including all orders in the exchange of photons between the target and the projectile. But the time evolution of the system during the excitation is neglected and only $E 1$ transitions are taken into account. The first approximation corresponds to an adiabaticity parameter $\xi$ of zero. This quantity is the ratio of the collision time to the nuclear interaction time and it is given by $\xi=\left(E_{0}+E_{\text {rel }}\right) b / \hbar v$. The multipole response of the system is characterized by effective charges $Z_{\text {eff }}^{(\lambda)}$ $=Z_{c}\left[m_{n} /\left(m_{n}+m_{c}\right)\right]^{\lambda}$. They become very small for higher multipolarities due to the small ratio $m_{n} /\left(m_{n}+m_{c}\right)$. From a perturbation expansion of the excitation amplitude it can be shown that the second order $E 1-E 1$ amplitude is also much larger than the first order $E 2$ amplitude. The ratio is given by the Coulomb (or Sommerfeld) parameter $Z Z_{c} e^{2} / \hbar v$ which is much larger than 1 for high charge numbers $Z$. Therefore we can safely neglect $E 2$ excitation in the following. (This is qualitatively different for, e.g., $p+$ core systems like ${ }^{8} \mathrm{~B}$ $\rightarrow{ }^{7} \mathrm{Be}+p$ with much larger $E 2$ effective charges.) This can be considered as a justification of the model of [8].

We expand the analytical results for the excitation probability of [8] for $\xi=0$ up to second order in $E 1$ excitation or equivalently in the characteristic strength parameter which is given by

$$
\chi=\frac{2 Z Z_{\mathrm{eff}}^{(1)} e^{2}}{v b \hbar k}
$$

where $k=\sqrt{\eta^{2}+q^{2}}$. In leading order (LO), the sudden limit of the first order result of [8] is obtained. Deviation for finite values of $\xi$ can be calculated according to [8]. From Eq. (12) of [8] one sees that the $\xi$ dependence of the amplitudes is given by $\xi K_{1}(\xi)$ or $\xi K_{0}(\xi)$ with modified Bessel functions, respectively. For $\xi=0$ this factor (for $K_{1}$ ) is 1 and drops to zero exponentially for $\xi \gg 1$. The $\xi$ correction in the next-toleading order (NLO) varies essentially like the square of this, so we can only overestimate the higher order effects in the present procedure.

Instead of using the strength parameter $\chi$ we define in the following the slightly different parameters

$$
y=\chi \frac{k}{\eta}
$$

(independent of $q$ ) and

$$
x=\frac{q}{\eta}=\sqrt{\frac{E_{\text {rel }}}{E_{0}}} .
$$


After angular integration over the relative momentum between the fragments the LO breakup probability is found to be [see Eq. (37) of [8]]

$$
\frac{d P_{L O}}{d q}=\frac{16}{3 \pi \eta} y^{2} \frac{x^{4}}{\left(1+x^{2}\right)^{4}}
$$

[Note that for a correct normalization of the breakup probability the results of [8] have to be divided by $(2 \pi)^{3}$.] The NLO contribution is proportional to $y^{4}$ and contains a piece from the second order $E 1$ amplitude and a piece from the interference of first and third order amplitudes. Again, in terms of the variables $\eta, x$, and $y$ one obtains

$$
\frac{d P_{N L O}}{d q}=\frac{16}{3 \pi \eta} y^{4} \frac{x^{2}\left(5-55 x^{2}+28 x^{4}\right)}{15\left(1+x^{2}\right)^{6}} .
$$

The LO expression is directly proportional to the $B(E 1)$-strength with its characteristic shape in the zerorange model. The NLO contribution will introduce a change of that shape. It is weighted most in collisions with the smallest possible impact parameters $b$ and can easily be evaluated. For $0.309<x<1.367$ the NLO contribution becomes negative with the largest reduction at a relative energy close to the binding energy. This is essentially due to the interference of first and third order amplitudes. The second order $E 1-E 1$ contribution is positive definite. From [8] we find

$$
\frac{d P_{E 1-E 1}}{d q}=\frac{16}{3 \pi \eta} y^{4} \frac{x^{2}\left(5+5 x^{2}+16 x^{4}\right)}{15\left(1+x^{2}\right)^{6}} .
$$

A reduction of the cross section at small relative energies is obtained only if third order contributions in the breakup amplitude are considered, in either a perturbative treatment (cf. Figs. 2-4 in [10]) or a full dynamical calculation (cf. Figs. 5 and 7 in [11]). In our analytical results we can directly see the dependence of higher order effects on the impact parameter $b$, the projectile velocity $v$, and the binding energy $E_{0}$ characterized by $\eta$. For larger impact parameters the first order $E 1$ contribution will dominate more and more $(y$ $\left.\propto b^{-1}\right)$. Perhaps experimental accuracy will not be high enough to see such a change of the shape of the breakup bump. The scaling variable $y$ also displays very clearly the dependence on the binding energy, characterized by $\eta$, and the charge number $Z$. Since $Z_{c} / m_{c}$ is approximately constant for all nuclei, the breakup probabilities for heavier nuclei (like the $r$-process nuclei) are expected to be of the same order of magnitude as for the light ones (like ${ }^{11} \mathrm{Be}$ or ${ }^{19} \mathrm{C}$ ). This will be an interesting field for future rare isotope accelerator (RIA) facilities, where intensive beams of medium energy neutron-rich nuclei will become available. This is of special interest for the $r$ process [12].

The breakup cross section can be obtained by multiplying the differential breakup probability by the Rutherford scattering cross section $d \sigma_{R} / d \Omega$ and the density of final states. It is given for the LO approximation by

$$
\frac{d^{2} \sigma_{L O}}{d E_{\mathrm{rel}} d \Omega}=\frac{d \sigma_{R}}{d \Omega} \frac{d P_{L O}}{d q} \frac{m}{\hbar^{2} q}
$$

and similarly for the NLO approximation. In order to have a quick estimate of higher order effects in the total breakup cross section we can integrate over the scattering angle and the breakup relative energy,

$$
\sigma=\int d E_{\mathrm{rel}} \int d \Omega \frac{d^{2} \sigma}{d E_{\mathrm{rel}} d \Omega}=2 \pi \int d q \int_{b_{\min }}^{b_{\max }} d b b \frac{d P_{L O}}{d q},
$$

where we have introduced minimum and maximum impact parameters $b_{\min }$ and $b_{\max }$, respectively. We use $b_{\max }$ $=\hbar v /\left(E_{0}+E_{\text {rel }}\right)$ corresponding to a cutoff at an adiabaticity parameter of $\xi=1$. The integration over the impact parameter $b$ is now easily performed. Introducing the effective strength parameter

$$
\chi_{\mathrm{eff}}=y \eta b=\chi k b=\frac{2 Z Z_{\mathrm{eff}}^{(1)} e^{2}}{\hbar v}
$$

and the minimal adiabaticity parameter

$$
\xi_{\min }=\frac{E_{0} b_{\min }}{\hbar v}
$$

we finally obtain

$$
\sigma_{L O}=\frac{\pi}{18}\left(\frac{\chi_{\mathrm{eff}}}{\eta}\right)^{2}\left[1-6 \ln \left(4 \xi_{\mathrm{min}}\right)\right]
$$

and

$$
\sigma_{N L O}=-\frac{\pi}{18} b_{\min }^{-2}\left(\frac{\chi_{\mathrm{eff}}}{\eta}\right)^{4}\left[\frac{23}{40}+18 \xi_{\min }^{2}\right]
$$

i.e., a reduction of the first order result. The total cross sections can only be a rough guide because of the simple treatment of the cutoff. Modifications due to a more precise treatment of the $\xi$ dependence usually have to be introduced [see below Eq. (3.1)]. However, the ratio gives a reasonable approximation to the higher order effects. It is a simple function depending on the characteristic parameters of the excited system and the experimental conditions.

In order to compare the results of the analytical model to a more realistic model we also perform fully dynamical calculations by solving the time-dependent Schrödinger equation for the evolution of the projectile system in the semiclassical approach. Here we have used the methods described in Ref. [13]. We refrain from a detailed presentation of the numerical technique and only give information specific to the actual calculation in this work. The wave function of the neutron-core system is expanded in partial waves where we take into account orbital angular momenta of $l=0, \ldots, 3$. We restrict ourselves to $E 1$ contributions in the multipole expansion of the perturbation potential as in the analytical model. The method of Ref. [13] has the virtue that both first and higher order calculations can be performed within the same 
approach by simply switching on and off the corresponding coupling potentials between the different partial waves. Within the method of Ref. [7] for the time evolution it is not obvious how to perform a first order calculation. However, we checked the correctness of the first order and fully dynamical calculations by comparing them to independently obtained results from the usual first order calculations and dynamical calculations using the technique of [7].

The wave function of the neutron-core system is discretized on a radial grid with maximum radius $R_{\max }$ $=900 \mathrm{fm}$ where the mesh points $x_{n}=n \Delta x$ are given in the same way as in [7] by the mapping $r_{n}=R_{\max }\left[\exp \left(a x_{n}\right)\right.$ $-1] /[\exp (a)-1]$ with $\Delta x=0.0025$ and $n=0, \ldots, 400$. The parameter $a$ is chosen to give $r_{1}=0.3 \mathrm{fm}$. We used a time step of $\Delta t=1 \mathrm{fm} / c$ in the time evolution and hyperbolic Coulomb trajectories. The distance between projectile and target for the start and end points of the calculation was determined by the condition that the perturbation potential was at least 200 times smaller than the value at closest approach. Additionally, the potential was switched on smoothly in order to avoid unphysical excitations. The final wave function is projected onto plane wave scattering states (after subtraction of contributions corresponding to bound states) in order to extract the excitation probabilities for a given c.m. energy in the neutron-core system.

\section{APPLICATION TO THE COULOMB BREAKUP OF ${ }^{19} \mathrm{C}$ AND ${ }^{11}$ Be}

In a recent experiment at RIKEN the breakup of ${ }^{19} \mathrm{C}$ into ${ }^{18} \mathrm{C}$ and a neutron scattered on a $\mathrm{Pb}$ target with a beam energy of $67 \mathrm{~A} \mathrm{MeV}$ was studied and the binding energy of the neutron was determined to be $0.53 \mathrm{MeV}$ [3]. We apply our model to this case since the high beam energy together with the simple structure and small binding energy of the neutron are favorable for a comparison.

In the dynamical model the neutron in the bound state of ${ }^{19} \mathrm{C}$ was assumed to be in a $2 s_{1 / 2}$ state with a binding energy of $0.53 \mathrm{MeV}$ as deduced by Nakamura et al. [3]. The wave function was calculated assuming a Woods-Saxon potential of radius $r=3.3 \mathrm{fm}$ and diffuseness parameter $a=0.65 \mathrm{fm}$. The depth is adjusted to $V=-39.77 \mathrm{MeV}$ in order to get the experimentally extracted binding energy. The ground state wave function obtained has a node in contrast to the zerorange model in the analytical calculation.

In Fig. 1 we show the double differential cross section as a function of the relative energy for three scattering angles. We have chosen $0.3^{\circ}, 0.9^{\circ}$, and $2.7^{\circ}$, which correspond to impact parameters of $109.7 \mathrm{fm}, 36.6 \mathrm{fm}$, and $12.2 \mathrm{fm}$, respectively. In order to compare the cross section in our analytical model with finite- $\xi$ results of the first order semiclassical calculation we multiply the analytical cross section given in Eq. (2.9) by the shape function

$$
\phi(\xi)=\xi^{2}\left[K_{0}^{2}(\xi)+K_{1}^{2}(\xi)\right]
$$

of the photon spectrum and a normalization factor $N$. The function $\phi(\xi)$ gives the correct dependence on the adiabaticity parameter in first order. We have $\phi(0)=1$ and it drops to
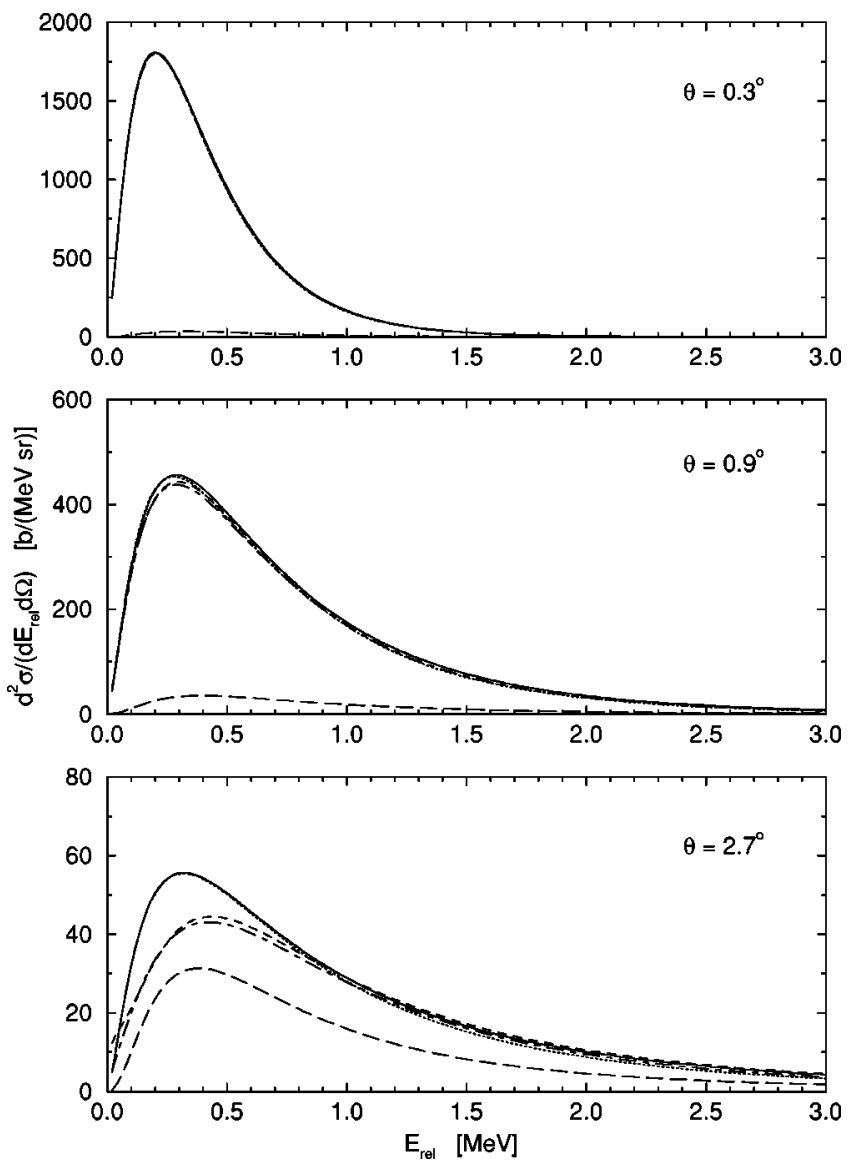

FIG. 1. Double differential cross section for the Coulomb dissociation of $67 \mathrm{MeV} /$ nucleon ${ }^{19} \mathrm{C}$ scattered on ${ }^{208} \mathrm{~Pb}$ as a function of the relative energy for three scattering angles. Analytical model with finite $\xi$ correction: LO calculation (solid line), LO+NLO calculation (dashed line). Semiclassical calculation: $E 1$ first order (dotted line), E1 dynamical (dot-dashed line), E2 first order multiplied by 1000 (long-dashed line).

zero rapidly for $\xi>1$. The factor $N$ accounts for finite-range effects. The ground state wave function in the analytical model is a $1 s_{1 / 2}$ state which has a different asymptotic normalization but the same slope as compared to the corresponding wave function from the Woods-Saxon potential. The slope of the wave function is determined by the binding energy [see Eq. (2.1)] which is the same in both models. The results of the dynamical calculation (dotted and dot-dashed lines) agree very well with the $\xi$-corrected cross section in the analytical model (solid and dashed lines) for $N=2.73$. This value is obtained by requiring the cross section in both models to be the same at the peak of the excitation function. This normalization factor is close to the value of $N=2.55$ resulting from a comparison of the asymptotic normalization of the two bound state functions. The small difference of the factors is caused by the different shapes of the wave functions inside the nuclear radius which give different contributions to the transition matrix element for finite relative energies in the continuum. There are noticeable differences between the analytical model and the dynamical calculation only for large relative energies and scattering angles. The first order E2 contribution (multiplied by 1000) is also 

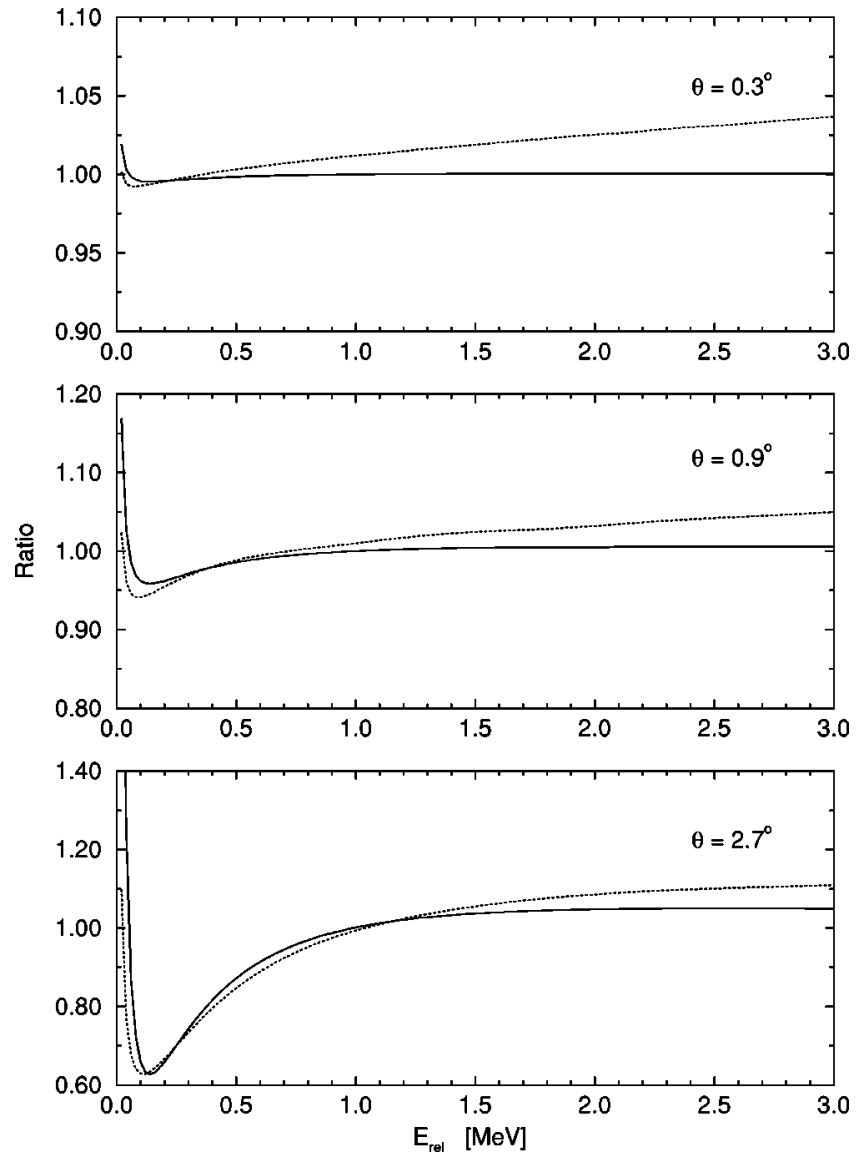

FIG. 2. Ratio of higher order to first order double differential cross sections for the Coulomb dissociation of $67 A \mathrm{MeV}{ }^{19} \mathrm{C}$ scattered on ${ }^{208} \mathrm{~Pb}$ as a function of the relative energy for three scattering angles. Analytical model with finite $\xi$ correction (solid line) and semiclassical calculation (dotted line).

shown in Fig. 1 (long-dashed line). It is at least 3 orders of magnitude smaller as compared to the first order $E 1$ excitation cross section and can safely be neglected. Furthermore, we observe that the cross section decreases strongly with increasing scattering angle. For small scattering angles the results of first and higher order calculations are almost identical. With increasing scattering angle we notice a reduction of the cross section for small relative energies due to higher order electromagnetic effects.

In Fig. 2 we compare the ratio of higher order (i.e., all orders in the dynamical calculation or $\mathrm{LO}+\mathrm{NLO}$ in the analyical model, respectively) to first order cross sections depending on the relative energy for the same scattering angles as in Fig. 1. The solid line gives the result of the analytical model. [Notice that the ratio is independent of $\phi(\xi)$ and $N$.] The dependence of the ratio on the relative energy again agrees well with the ratio in the full semiclassical model (dotted line). At small relative energies there is a reduction of the cross section (except for energies close to zero) whereas at higher relative energies we find a small increase. This behavior can be understood directly by inspecting Eqs. (2.6) and (2.7). Higher order effects are largest for large scattering angles corresponding to impact parameters close to grazing scattering. A look at the breakup probabilities (2.6) and (2.7)
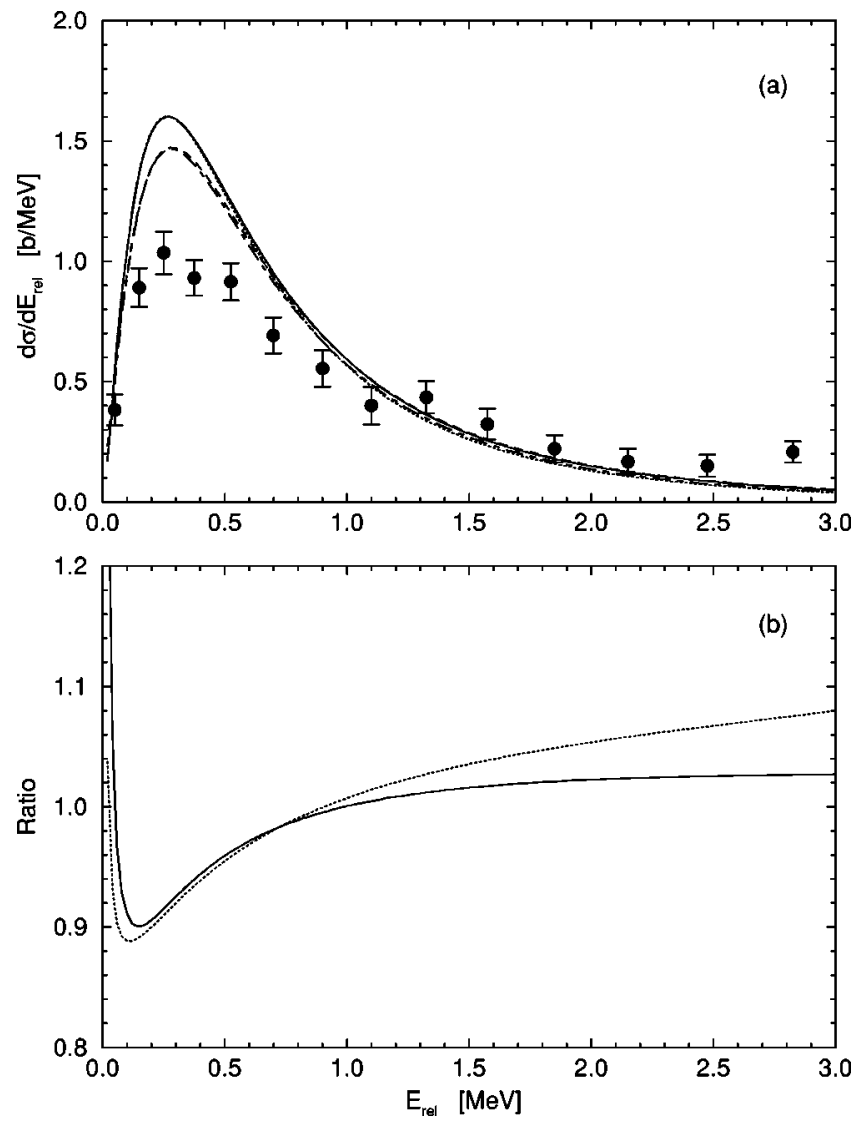

FIG. 3. (a) Differential cross sections integrated over scattering angle from $0^{\circ}$ to $3^{\circ}$ for the Coulomb dissociation of $67 \mathrm{~A} \mathrm{MeV}{ }^{19} \mathrm{C}$ scattered on ${ }^{208} \mathrm{~Pb}$ as a function of the relative energy. Analytical model with finite $\xi$ correction: LO calculation (solid line), LO+NLO calculation (dashed line). Semiclassical calculation: $E 1$ first order (dotted line), E1 dynamical (dot-dashed line). Experimental data from [3]. (b) Ratio of higher order to first order differential cross sections for the $\xi$-corrected analytical model (solid line) and the dynamical model (dotted line).

shows that higher order effects increase essentially with $b^{-2}$. The discrepancy between the two models at higher relative energy, where the exact form of the wave function for small radii in the range of the nuclear potential becomes important, is not essential because the absolute cross sections are very small. In contrast, at small relative energies, the models agree very well since the main contribution to the matrix elements is determined by the asymptotic form of the wave function.

Integrating the double differential cross sections over scattering angles between $0^{\circ}$ and $3^{\circ}\left(b_{\min } \approx 11 \mathrm{fm}\right)$ leads to the energy-dependent cross sections in Fig. 3(a). Again we observe that both the first order and higher order calculations in the $\xi$-corrected analytical model and the full semiclassical model agree very well at the peak of the distribution. Here we find a reduction of the cross section of at most $10 \%$ at small relative energies as shown in Fig. 3(b). The spectral shape is not severely distorted. Higher order Coulomb effects cannot explain the difference between first order theoretical calculations and the experimental results with respect to the absolute magnitude and the shape of the experimental data 
[3]. Although they lead to a decrease of the cross section at small energies (apart from the region just above threshold) and an increase at higher energies the slope of the theoretical results is much steeper as compared to the experiment. In contrast, the position of the peak is well described since it is determined by the binding energy of the neutron in ${ }^{19} \mathrm{C}$.

By integrating over relative energies the effects of higher order are washed out and become even smaller in the total cross section $\sigma$. We obtain $1.44 \mathrm{~b}(1.39 \mathrm{~b})$ in the first order (dynamical) semiclassical calculation and $1.49 \mathrm{~b}(1.44 \mathrm{~b})$ in the LO (NL+NLO) analytical model with $\xi$ correction, respectively, for energies up to $3 \mathrm{MeV}$. Comparing to the experimental value of $\sigma=1.34 \pm 0.12 \mathrm{~b}$ [3] one has to take into account our simple nuclear model. In reality the ground state of ${ }^{19} \mathrm{C}$ has a more complicated structure than a $2 s_{1 / 2}$ single particle state. Multiplying the cross sections of our calculation by a spectroscopic factor of 0.67 as given by Nakamura et al. [3] the total cross section would be smaller than in the experiment but the peak region in Fig. 3(a) would be well described. At higher relative energies nuclear contributions could be present in the experimental data, increasing the total cross section again. Possible Coulomb-nuclear interference effects could also lead to a change of shape of the cross section. Furthermore, the experimental data could contain contributions from final states with an excitation of the core ${ }^{18} \mathrm{C}$. Our results correspond to a reduction of the total cross section by higher order effects of $3.3 \%$ in the semiclassical model and of $3.2 \%$ in the $\xi$-corrected analytical model. From Eqs. (2.13) and (2.14) we predict a $2.9 \%$ reduction which is close to the results of more refined models. From the comparison we conclude that our simple analytical model with finite- $\xi$ correction is quite realistic in the prediction of higher order effects and gives a reliable estimate of the reduction of the total cross section. Finite-range effects amount essentially to a rescaling of the $B(E 1)$ matrix element and, correspondingly, the cross section. The smaller value of the reduction obtained in the simpler fully analytical model can be well understood. Without taking the adiabatic suppression correctly into account contributions to the total cross section from higher relative energies and larger impact parameters, where higher order effects are smaller, are not sufficiently reduced and lead to an underestimate. However, higher order effects in the triple differential cross section in the peak of the excitation function are well described by the simple analytical expressions. The influence of higher order effects on the cross section seems to be rather independent of the interior wave function for the $n$-core system. It is essentially determined by the asymptotic wave function for a halo nucleus. This can be well understood in a classical picture where the interaction of the fragments with the target has a stronger effect on the relative momentum the larger their distance becomes. It is also reflected in perturbative calculations of higher order effects by the appearance of transition operators that contain an $r^{\lambda}$ dependence where $\lambda$ increases with the order, leading to an emphasis on the asymptotic wave function.

Our results are in contrast to those of [6] where a much bigger effect of the order of 30 to $40 \%$ was found by comparing a first order semiclassical result with the fully quantal

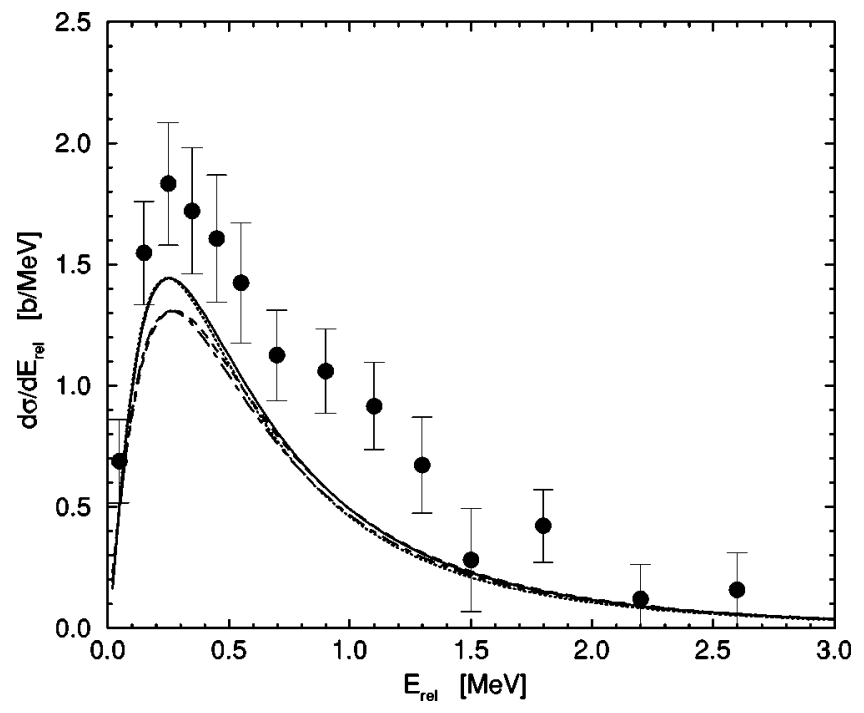

FIG. 4. Differential cross sections integrated over scattering angle from $0^{\circ}$ to $3^{\circ}$ for the Coulomb dissociation of $72 \mathrm{~A} \mathrm{MeV}$

${ }^{11} \mathrm{Be}$ scattered on ${ }^{208} \mathrm{~Pb}$ as a function of the relative energy. Analytical model with finite- $\xi$ correction: LO calculation (solid line), LO+NLO calculation (dashed line). Semiclassical calculation: $E 1$ first order (dotted line), E1 dynamical (dot-dashed line). Experimental data from [2].

adiabatic approach in the finite-range case for the neutroncore interaction. On the other hand much smaller effects were obtained with the zero-range model in [6]. It is difficult to assess how much of the reduction of the cross section is caused essentially by higher order electromagnetic effects or by differences between these models. However, in both cases, the total contribution of higher orders to the cross section is negative.

Let us now consider the case of ${ }^{11} \mathrm{Be}$ breakup at $72 \mathrm{~A} \mathrm{MeV}$ where essentially the same considerations apply as in the ${ }^{19} \mathrm{C}$ case. In view of the simple scaling laws presented in Sec. II [see especially Eqs. (2.4)-(2.7)] this is to be expected. We assume that the neutron in ${ }^{11} \mathrm{Be}$ is bound by $0.503 \mathrm{MeV}$ [2]. The $2 s_{1 / 2}$ ground state wave function in the dynamical finite range model was calculated from a WoodsSaxon potential of $V=-69.79 \mathrm{MeV}$ with a radius of $r$ $=2.478 \mathrm{fm}$ and a diffuseness parameter of $a=0.5 \mathrm{fm}$. Additionally, we included a bound $1 p_{1 / 2}$ state at $0.320 \mathrm{MeV}$, which corresponds to a potential depth of $V$ $=-37.52 \mathrm{MeV}$. The cross sections in the analytical zerorange model were again corrected for finite- $\xi$ effects and multiplied by a normalization factor of $N=1.58$. The differential cross section $d \sigma / d E_{\text {rel }}$ was calculated as in the ${ }^{19} \mathrm{C}$ case by an integration of the double differential cross section over the scattering angle up to $3^{\circ}$ corresponding to a minimum impact parameter of $b=11.8 \mathrm{fm}$. In Fig. 4 we compare the LO and NLO results in the analytical model with the first and higher order results in the dynamical model. We observe the same features as in the ${ }^{19} \mathrm{C}$ case. The first order calculations and the higher order results in both models look very similar at the peak of the excitation function. In both cases we obtain a reduction of the cross section in the peak region by less than $10 \%$. This agrees with the reduction of the ${ }^{11} \mathrm{Be}$ 
breakup cross section by higher order effects found by the authors of Ref. [11] who employed a similar potential model and dynamical approach. Integrating over the energy between 0 and $3 \mathrm{MeV}$ the reduction of the total cross sections by higher order effects is found to be $-3.6 \%$ in the $\xi$-corrected analytic model and $-3.2 \%$ in the dynamical calculation. The reduction of $-4.1 \%$ deduced from Eqs. (2.13) and (2.14) is a little larger than the results from the more refined calculations. Contrary to the ${ }^{19} \mathrm{C}$ case the calculated cross sections are lower than the experimental data [2]. The same result is obtained in the nonperturbative calculation of Ref. [7]. The small difference in the overall normalization of the calculated cross section is probably related to the slighly different nuclear model of ${ }^{11} \mathrm{Be}$ and the use of straight-line trajectories. The authors of Ref. [7] compare their result only to another partly nonperturbative, partly perturbative calculation [14] but not to a first order calculation with the same nuclear model. As a consequence, they do not make statements about the size of electromagnetically induced higher order effects in the strict sense.

Finally, let us make some remarks about postacceleration. A semiclassical model might suggest that the parallel momentum distribution of the core is shifted toward larger values due to an "extra Coulomb push," see, e.g., [9]. However, this turns out to be wrong. In the sudden approximation, the core-neutron binding is negligible and also, on its way toward the target, the core alone (and not the bound coreneutron system) experiences the Coulomb deceleration. Formally, this is easily seen: In the sudden approximation, the momentum transfer points exactly to the direction perpendicular to the trajectory, the excitation amplitude depending only on $\vec{q} \cdot \Delta \vec{p}$ (cf. [8]). This is symmetric with respect to the plane perpendicular to the beam direction. Corrections of this simple result due to small values of $\xi$ were studied in [8]. They were found to depend only on the phase shift of the neutron $s$ wave. This phase shift is given in the analytical model by $\delta_{0}=-\arctan (q / \eta)$. It is a rather delicate quantal interference effect and even has the opposite sign to what one would have thought "intuitively." Large values of $\xi$ correspond to large values of $b$ where the strength parameter is small. Therefore, higher order effects are not so important. Indeed, in Ref. [15] no effects of postacceleration were found for the ${ }^{11} \mathrm{Be}$ system.

\section{CONCLUSIONS}

We have studied the basic example for the Coulomb dissociation of a neutron halo nucleus. From the simple zerorange wave function of a loosely bound system it becomes directly obvious that the low lying $E 1$ strength is an immediate consequence of the halo structure. It is probably the most beautiful manifestation of the halo nature. The reaction mechanism is now understood at such a quantitative level that it is possible to determine asymptotic normalization coefficients with the Coulomb dissociation method to a high accuracy. Higher order effects can be described by analytical formulas. This allows a very transparent discussion of the effects. Our results can be easily applied to all neutron halo Coulomb dissociation experiments. They are a useful guide for the much more elaborate numerical solutions of the timedependent Schrödinger equation. Our simple considerations are corroborated by these more sophisticated approaches. We conclude that higher order electromagnetic effects are not a significant problem in medium energy Coulomb dissociation experiments and can be kept under control.

\section{ACKNOWLEDGMENTS}

The authors would like to thank R. Shyam, B. A. Brown, and P. G. Hansen for useful discussions and for helpful remarks on the manuscript. We are grateful to T. Nakamura for providing us with the experimental data. Support for this work was provided by the U.S. National Science Foundation Grant Nos. PHY-0070911 and PHY-9528844.
[1] R. Anne et al., Phys. Lett. B 304, 55 (1993); Nucl. Phys. A575, 125 (1994).

[2] T. Nakamura et al., Phys. Lett. B 331, 296 (1994).

[3] T. Nakamura et al., Phys. Rev. Lett. 83, 1112 (1999).

[4] G. Baur, K. Hencken, D. Trautmann, S. Typel, and H. Wolter, in Spins in Nuclear and Hadronic Reactions, Proceedings of the RCNP-TMU Symposium, edited by H. Yabu, T. Suzuki, and H. Toki (World Scientific, Singapore, 2000), p. 119.

[5] J. A. Tostevin, S. Rugmai, and R. C. Johnson, Phys. Rev. C 57, 3225 (1998).

[6] J. A. Tostevin, in Spectroscopy of Halo Nuclei From Reaction Measurements, Proceedings of the Second International Conference on Fission and Properties of Neutron-Rich Nuclei, St. Andrews, Scotland, 1999, edited by J. H. Hamilton, W. R. Phillips, and H. K. Carter (World Scientific, Singapore, 2000), p. 429.

[7] V. S. Melezhik and D. Baye, Phys. Rev. C 59, 3232 (1999).
[8] S. Typel and G. Baur, Nucl. Phys. A573, 486 (1994).

[9] G. Baur, C. A. Bertulani, and D. M. Kalassa, Nucl. Phys. A550, 527 (1992).

[10] S. Typel, G. Baur, and H. H. Wolter, Nucl. Phys. A613, 147 (1997).

[11] H. Esbensen, G. F. Bertsch, and C. A. Bertulani, Nucl. Phys. A581, 107 (1995).

[12] G. Baur, K. Hencken, D. Trautmann, S. Typel, and H. H. Wolter, Radioactive Beams for Nuclear and Astrophysics, Proceedings of the International Workshop of Nuclear Physics, 22nd Course, Erice, Sicily, 2000 [Prog. Part. Nucl. Phys. 46, 99 (2001)].

[13] S. Typel and H. H. Wolter, Z. Naturforsch., A: Phys. Sci. 54, 63 (1999).

[14] T. Kido, K. Yabana, and Y. Suzuki, Phys. Rev. C 53, 2296 (1996).

[15] J. E. Bush et al., Phys. Rev. Lett. 81, 61 (1998). 